\title{
Nalfurafine hydrochloride for refractory pruritus in peritoneal dialysis patients: a phase III, multi-institutional, non-controlled, open-label trial
}

Hidetomo Nakamoto' ${ }^{1}$ Takanori Oh², Masahiro Shimamura², Eiji lida ${ }^{2^{*}}$ and Sadanobu Moritake ${ }^{3}$

\begin{abstract}
Background: Nalfurafine hydrochloride ("nalfurafine"), the world's first selective oral k-receptor agonist for improving pruritus, is approved in Japan for the treatment of pruritus resistant to existing treatments in hemodialysis (HD) or chronic liver disease patients. Peritoneal dialysis (PD) patients, like HD patients, suffer from end-stage renal disease (ESRD) and some experience refractory pruritus.

Methods: We investigated the efficacy and safety of nalfurafine in 37 ESRD patients who underwent PD and had refractory pruritus. Nalfurafine was given once daily for 4 weeks at $2.5 \mu \mathrm{g}$ in weeks 1 and 2 of the treatment period and at $5 \mu \mathrm{g}$ in weeks 3 and 4 . The primary endpoint was visual analog scale (VAS) changes for pruritus (i.e., the value upon rising or before sleep in week 2, whichever larger).

Results: The mean VAS change from baseline in week 2 of the treatment period was $24.93 \mathrm{~mm}[18.67,31.19]$ (the point estimate of the mean [90\% confidence interval (CI)]); the lower limit of $\mathrm{Cl}$ exceeded the point estimate of the mean VAS change $(15.24 \mathrm{~mm}$ ) of the placebo group at the evaluation point (week 2) in a preceding confirmatory trial suggesting that had demonstrated nalfurafine efficacy for refractory pruritus in HD patients. The observed VAS change was comparable to that of the $2.5-\mu \mathrm{g}$ group (week 2 ) in the preceding confirmatory trial, demonstrating that nalfurafine is as effective for treating pruritus in PD patients as in HD patients. Nalfurafine $5 \mu \mathrm{g}$ was associated with a mean VAS change of $32.13 \mathrm{~mm}$ at week 4, i.e., the full length of the trial treatment period suggesting efficacy at the dose of $5 \mu \mathrm{g}$. The incidence of adverse drug reactions (ADR) was 45.9\% (1/37 patients) with no serious ADRs observed. ADRs occurring in $\geq 5 \%$ of patients included insomnia (13.5\%), increased blood prolactin (13.5\%), somnolence (8.1\%), lower blood testosterone free (8.1\%), and vomiting (5.4\%), all of which were mild.
\end{abstract}

Conclusions: This trial demonstrated the efficacy and safety of nalfurafine against refractory pruritus in PD, suggesting clinical benefit for treating pruritus in PD patients.

Trial registration: Japan Pharmaceutical Information Center, JapicCTI-142565

Keywords: Nalfurafine, k-receptor agonist, Peritoneal dialysis, Pruritus, Refractory, Visual analog scale, Phase III trial

\footnotetext{
*Correspondence: Eiji_lida@nts.toray.co.jp

${ }^{2}$ Pharmaceutical Clinical Research Department, Toray Industries Inc., 1-1,

Nihonbashi-muromachi 2-chome, Chuo-ku, Tokyo 103-8666, Japan

Full list of author information is available at the end of the article
} 


\section{Background}

Peritoneal dialysis (PD) patients suffering from end-stage renal failure, as with hemodialysis (HD) patients, often experience pruritus manifesting as itch $[1,2]$. Itch may accompany diseases such as skin diseases (urticaria, atopic dermatitis, etc.), liver diseases (primary biliary cholangitis, etc.), renal diseases (chronic renal failure, etc.), and endocrine disorders (diabetes, thyroid dysfunction, etc.) [3]. Skin itch in patients with urticaria, for which is largely induced by locally existing mediators such as histamine, can be controlled by antihistamines or other anti-allergy treatment. In contrast, renal disease-induced pruritus in PD patients (in common with HD patients) is often highly resistant to current treatments. The unmet medical need to establish a treatment with a useful agent for such patients is, therefore, pressing [1-3].

Nalfurafine hydrochloride (nalfurafine) is a novel selective $\kappa$-receptor agonist developed by the Pharmaceutical Research Laboratories of Toray Industries, Inc. In non-clinical studies, nalfurafine showed an antipruritic effect for an itch model in which antihistamine was not sufficiently effective [4, 5]. Based on this finding, clinical development of an oral agent was launched for the treatment of refractory pruritus in HD patients [6, 7]. Following confirmation of its clinical effectiveness, nalfurafine was approved for use in Japan in 2009 for the indication of improving refractory pruritus in HD patients. Nalfurafine efficacy for intractable pruritus in patients with chronic liver diseases (hepatitis, hepatic cirrhosis, primary biliary cholangitis, non-alcoholic steatohepatitis, etc.) was subsequently confirmed during clinical development of the agent [8]. Additional approval was consequently obtained in 2015 for the indication of improving refractory pruritus in patients with chronic liver disease.

In common with HD patients, pruritus in PD patients typically starts prior to the introduction of dialysis during the preservation period of chronic renal failure; no major differences have been observed in the incidence and severity of pruritus between these populations $[1,2,9]$. As the fundamental mechanism of PD is the same as that of HD (the exception being that PD is performed via the patient's peritoneum), pruritus may be attributed to chronic renal failure both in PD and HD patients, with endogenic opioids playing a role in its onset [10]. Given these features, it is hypothesized that nalfurafine could be as effective for refractory pruritus in PD patients as in HD patients.

The results of preceding clinical pharmacological studies indicated that there is no requirement for adjustment of the nalfurafine according to the different prescriptions of PD (unpublished observations). These earlier studies also suggest that the pharmacokinetics of nalfurafine is similar in PD and HD patients.
On the basis of these findings, the present trial was conducted to investigate the safety and efficacy (i.e., the ability to provide additional clinical benefit over the base treatment of pruritus) of nalfurafine administered to PD patients with refractory pruritus with the same dosage and regimen as in the preceding confirmatory trial in HD patients. This trial was registered on June 5, 2014 to Japan Pharmaceutical Information Center (JAPIC) as JapicCTI-142,565.

\section{Methods}

This open-label investigation of nalfurafine therapy added to a base treatment against pruritus was conducted at 25 institutions in Japan from August 2014 to June 2015. In consideration of the small number of PD patients in Japan with approximately 9300 patients [11], no control group was included. Instead, the observed results were compared to those of a preceding confirmatory trial in which nalfurafine was tested against pruritus resistant to existing treatments in HD patients [6]. Therefore, for the purpose of aligning patient characteristics as much as possible between the trials, virtually the same inclusion and exclusion criteria as the preceding confirmatory trial in HD patients were employed. The same evaluation criteria for efficacy were similarly adopted. The trial schedule consisted of a 2-week pretreatment period, a 4-week treatment period, and an 8-day follow-up period (Fig. 1).

During the pretreatment period, we observed the level of pruritus under base treatment alone to determine the baseline value. In weeks 1 and 2 of the treatment period, the additional clinical benefit of nalfurafine $2.5 \mu \mathrm{g}$ over base treatment efficacy was determined. In weeks 3 and 4 of the treatment period, the dosage was increased from $2.5 \mu \mathrm{g}$ to $5 \mu \mathrm{g}$ and the added clinical benefit of nalfurafine was investigated.

This clinical trial obtained approval from each institutional review board of all the participating institutions. It was conducted in compliance with the ethical principles based on the Declaration of Helsinki, Pharmaceutical and Medical Device Act, and Ministerial Ordinance on Good Clinical Practice (J-GCP). Informed consent was obtained from all patients.

\section{Trial population}

Table 1 shows key inclusion criteria and exclusion criteria. Inclusion criteria 2 and 5 were set to ensure the time for patients to acclimatize to the PD manipulation and achieve a stable condition. Inclusion criteria 3 and 4 were set for the purpose of enrolling patients with pruritus refractory to existing treatments. The aim of inclusion criteria 6 and 8 were to confirm that the patient was not responding to the "supposedly most effective" base treatment, using the two pruritus endpoints (i.e., 


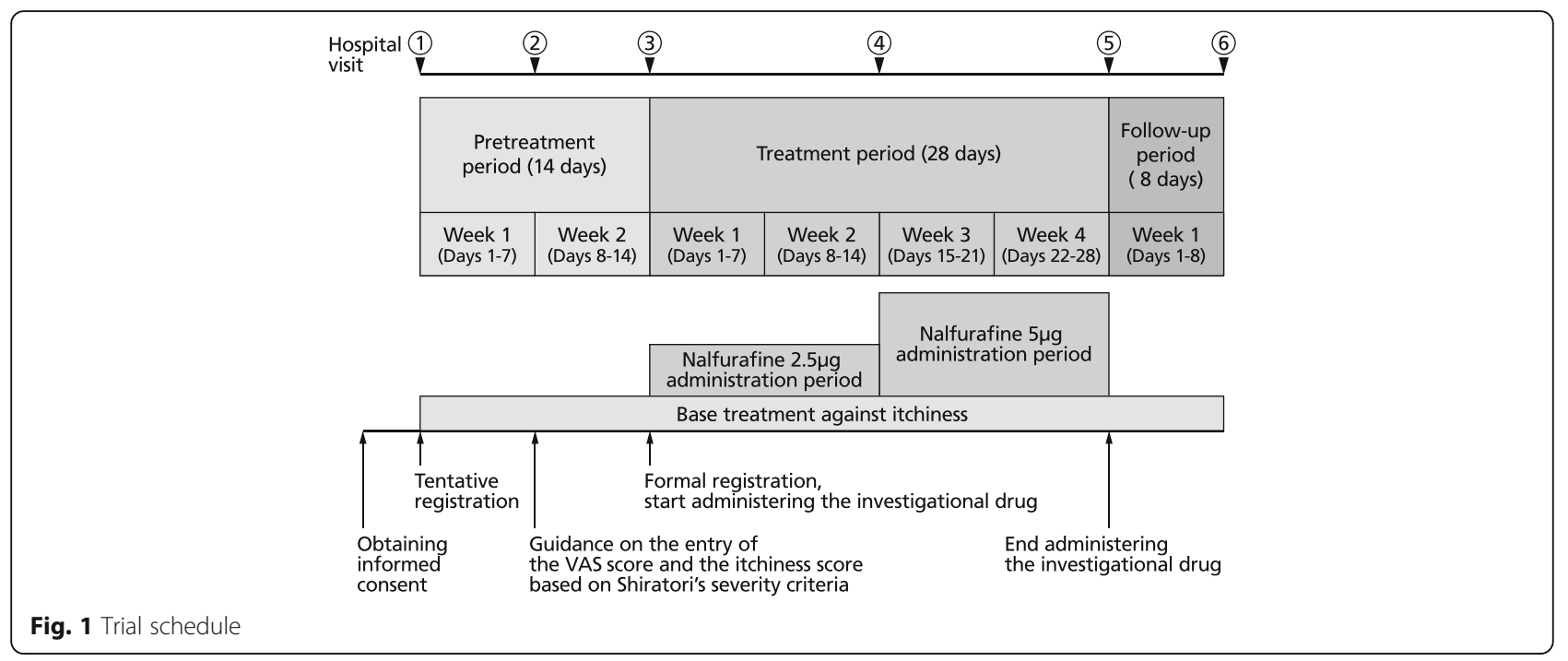

the VAS [12] and the Shiratori's criteria of pruritus severity [13]). In order to identify the severity and condition of pruritus, enrolled patients were required to have had the pruritus response endpoints recorded for five or more out of the 7 days during week 2 in the pretreatment period. Moreover, in order to enroll only those patients with severe pruritus and notable conditions sufficient for the assessment of pruritus, an inclusion criterion was established to require the severity and condition of pruritus in week 2 in the pretreatment period to be moderate or worse. Specifically, included patients had, on over half of the days, "the mean VAS of $50 \mathrm{~mm}$ or higher" and "the pruritus score of 3 or higher." Furthermore, inclusion criterion 7 was set to ensure the inclusion of patients with at least a certain level of constant pruritus. Exclusion criteria were established in order to eliminate any influence on the evaluation of efficacy and safety of nalfurafine.

\section{Treatment}

Previously used pruritus medical treatments were carried over as base treatment when a participant had either been treated prior to visit 1 with a prescription drug indicated for the treatment of pruritus, an over-thecounter (OTC) drug indicated for the treatment of pruritus, moisturizer, or an in-hospital preparation consisting of one or more of the above. In case a patient had received no pruritus medical treatment prior to visit 1 , the most likely effective agent was chosen from the pruritus treatment drugs used within 1 year before the informed consent acquisition date. Enrolled patients received oral nalfurafine once daily after evening meals $2.5 \mu \mathrm{g}$ [ 1 capsule] in weeks 1 and 2 in the treatment period and $5 \mu \mathrm{g}$ [ 2 capsules] in weeks 3 and 4 in the treatment period. Use of opioid drugs was prohibited during clinical trial due to the concern that they might enhance or reduce the effect of nalfurafine. The type, dosage, and regimen of prescription drugs and OTC drugs indicated for the treatment of pruritus remained unchanged, and newly initiating the use of the same drugs were prohibited to avoid their influence and allow adequate evaluation of pruritus during clinical trial. Specific designated drugs (i.e., hypnotics, antidepressants, antipsychotics, antiepileptics, anxiolytics, and non-prescription hypnotics) were also restricted due to the potential for increased adverse effects on the central nervous system when combined with nalfurafine.

\section{Efficacy assessment}

The severity and change of pruritus were evaluated by the VAS and the pruritus score based on Shiratori's criteria of pruritus severity $(0=$ no symptoms, $1=$ slight, $2=$ mild, 3 = moderate, $4=$ severe) [13]. Both assessment methods had been used in a preceding confirmatory trial measuring pruritus in HD patients [6].

Participants recorded the severity and change of pruritus each day from the first day of the pretreatment period to the last day of the follow-up period; each patient recorded the severity of worst pruritus upon rising and before bed every day. The primary endpoint was mean VAS change (determined by using the larger value recorded either upon rising or before bed) in week 2 of the treatment period, with missing data imputed by the last observation carried forward (LOCF). A threshold was set at $15.24 \mathrm{~mm}$, which derives from the point estimate of the mean change in the VAS (calculated using the larger value recorded either upon rising or before bed) of the placebo group in week 2 during the treatment period in the confirmatory trial enrolling HD patients. It was determined that nalfurafine was effective against refractory pruritus in PD patients when the lower limit of the $90 \%$ confidence interval (CI) of the 
Table 1 Eligibility criteria

Key inclusion criteria
Included patients had to meet all of the conditions listed below,
all sexes and both inpatients and outpatients were eligible.
Upon obtaining informed consent
1. A patient of age 20 years or older
2. A patient undergoing peritoneal dialysis for three consecutive
months or longer
3. A patient with a history within 1 year of drug treatment (a) and
(b) against itch
(a) systemic treatment (oral, injection, etc.) for two consecutive
weeks or longer using antihistamine or antiallergic drugs
indicated for the treatment of itch
(b) topical treatment (external, etc.) using ointment indicated
for the treatment of itch or moisturizer prescribed by a
physician
4. A patient for whom neither of the treatment against itch listed
in criteria 3 was evaluated sufficiently effective by the principal
investigator or another investigator
Upon formal registration
5. A patient undergoing peritoneal dialysis continuously from the
date of informed consent to the date of formal registration
6. A patient whose VAS score was recorded both on rising and
before bed for five or more days in week 2 in pretreatment
period, and the mean of the larger VAS score either upon
rising or before bed was 50 mm or larger
7. A patient of whom the larger VAS score either upon rising or
before bed was 20 mm or larger for five or more days in
week 2 of the pretreatment period
8. A patient whose itch score based on Shiratori's severity criteria
was recorded both on rising and before bed for 5 days or more
in week 2 in the pretreatment period, and the itch score either
upon rising or before bed, whichever was score of 3 or higher (i.e.,
moderate or worse), for half of the days

Key exclusion criteria

Patients were excluded from the study if one or more of the following conditions applied.

Upon obtaining informed consent

1. A patient who is concurrently undergoing hemodialysis

2. A patient who has been clinically diagnosed with the following complications

(a) encapsulating peritoneal sclerosis

(b) atopic dermatitis, chronic urticarial, or other skin disease that was determined to develop a systemic itch symptom that affects the evaluation of itch induced by renal disease

3. A patient who developed peritonitis within the last 4 weeks to make it difficult to continue peritoneal dialysis

4. A patient who received a phototherapy against itch in the last 1 month

5. A patient who has received nalfurafine in the past, who has participated in a clinical trial of nalfurafine and received it as the investigational drug, or who already participated as an eligible patient in this study

Upon formal registration

6. A patient who underwent hemodialysis between the date of informed consent and the date of formal registration

7. A patient who experienced onset of one of the following diseases between the date of informed consent and the date of formal registration

(a) encapsulating peritoneal sclerosis

(b) atopic dermatitis, chronic urticaria, or other skin disease that was determined to develop a systemic itch symptom that affects the evaluation of itch induced by renal disease

8. A patient who developed peritonitis between the date of informed consent and the date of formal registration, which made it difficult to continue peritoneal dialysis

9. A patient who received a phototherapy against itch between the date of informed consent and the date of formal registration mean change in the VAS of a PD patient was larger than the threshold value. We also evaluated as secondary endpoints the mean change in the VAS, the mean change in the pruritus score, and the VAS improvement during each evaluation period (i.e., in weeks 1, 2, 3, and 4 in the treatment period and week 1 in the follow-up period); no imputation using LOCF was performed. The mean VAS change in each evaluation period was calculated by subtracting the mean VAS of each period from the mean VAS in week 2 of the pretreatment period. The change in pruritus score was calculated similarly. The VAS improvement was considered effective when the mean VAS change in each evaluation period was $20 \mathrm{~mm}$ or larger and considered ineffective if smaller than $20 \mathrm{~mm}$ [14]. We also assessed the mean VAS changes in week 2 (LOCF) of the treatment period in subgroups categorized according to demographic characteristics or other reference values.

\section{Safety assessment}

Adverse events (AEs) reported from the initiation of the treatment period to the end of the follow-up period were investigated. Incidents of adverse drug reactions (ADRs), which excluded AEs with no causal relations with the investigational drug, were also recorded. Laboratory values, vital signs, and incidents of ECG abnormalities were also included in the assessment. The data were aggregated for each of the following four periods: from the start of nalfurafine $2.5-\mu \mathrm{g}$ administration to the end of the follow-up period (treatment period + follow-up period), from the start of $2.5-\mu \mathrm{g}$ administration to its termination before switching to $5 \mu \mathrm{g}(2.5-\mu \mathrm{g}$ administration period), from the start of $5-\mu \mathrm{g}$ administration to the first day of the follow-up period (5- $\mu$ g administration period), and from the start of 5- $\mu \mathrm{g}$ administration to the end of the follow-up period (5- $\mu \mathrm{g}$ administration period + follow-up period). Once the 5- $\mu$ g administration started, AEs were aggregated and analyzed only if the event had newly developed, or the severity had increased, after receiving $5 \mu \mathrm{g}$ of nalfurafine. If the change from the value obtained before the start of the 5 - $\mu$ g administration was considered clinically abnormal, those changes in laboratory values and vital signs were considered to indicate AEs.

\section{Statistical analysis}

The efficacy analysis primarily targeted the full analysis set (FAS). The primary endpoint was represented by the point estimate and the two-sided $90 \% \mathrm{CI}$ of the mean VAS change. Regarding secondary endpoints, the VAS change and the pruritus score change were shown by the fundamental statistics with two-sided 95\% CIs; meanwhile, the VAS improvement was computed as the point estimate of the improvement rate with two-sided 
95\% CIs. The incidence of AEs and ADRs were counted in accordance with the MedDRA/J (version 18.0) preferred terms. The analyses were conducted by using SAS software version 9.3 (Cary, NC, USA).

\section{Results}

Patients

Informed consent was obtained from 44 patients, of whom 37 met the eligibility criteria and formally registered. In total, 37 patients received nalfurafine $2.5 \mu \mathrm{g}$. However, two patients dropped out due to AEs (one patient each for drug eruption and hypoaesthesia/ hypoaesthesia oral/feeling hot/dizziness/vomiting); they did not receive nalfurafine $5 \mu \mathrm{g}$. The remaining 35 patients completed the entire course of nalfurafine $2.5 \mu \mathrm{g}$ regimen followed by nalfurafine $5 \mu \mathrm{g}$. Table 2 shows the demographic characteristics of the formally registered patients. The residual "other primary diseases" category consisted of three for renal sclerosis, four for congenital cystic kidney disease, and one for benign prostatic hyperplasia (including patients with multiple diseases).

\section{Efficacy}

The FAS comprised all 37 patients enrolled in the trial. The FAS analysis showed the efficacy of nalfurafine $2.5 \mu \mathrm{g}$ against pruritus resistant to existing treatments in PD patients as follows: the primary endpoint, that is, the mean VAS change in week 2 (LOCF) of the treatment period, was $24.93 \mathrm{~mm}$ [18.67, 31.19] (point estimate of the mean $[90 \% \mathrm{CI}]$ ) and the lower limit of the CI was larger than the threshold $(15.24 \mathrm{~mm})$. Increased VAS changes were observed during the treatment period, while the VAS changes declined after administration was completed. The VAS change in each of the evaluation periods was $16.71 \mathrm{~mm}[10.11,23.31]$ (point estimate of the mean [95\% CI]), $24.02 \mathrm{~mm}[16.52,31.53], 28.94 \mathrm{~mm}$ [21.38, 36.50], $32.13 \mathrm{~mm}[24.08,40.18]$, and $19.48 \mathrm{~mm}$ $[14.10,24.85]$ in weeks $1,2,3$, and 4 of the treatment period and in week 1 of the follow-up period, respectively (Fig. 2a). The course of the pruritus score change was similar to that of the VAS change. The pruritus score change in each of the evaluation periods was 0.61 [0.39, 0.83], 0.80 [0.52, 1.07], 0.97 [0.72, 1.22], 1.09 [0.80, $1.37]$, and $0.62[0.40,0.84]$ in weeks $1,2,3$, and 4 of the treatment period and in week 1 of the follow-up period, respectively (Fig. 2b). The VAS improvement was continuously enhanced in weeks 1 through 3 and then sustained in weeks 3 and 4 of the treatment period and diminished after the end of administration (Table 3). No background factors were found to have influenced the efficacy of nalfurafine; none of the subgroups categorized by demographic characteristics or other reference values showed any specific tendencies in the subgroup analysis of the VAS change in week 2 in the treatment period.
The difference in PD prescriptions, including the type of dialysate solution and PD, had virtually no influence on the efficacy of the agent. The mean VAS change was $25.11 \pm 20.65 \mathrm{~mm}$ (mean $\pm \mathrm{SD}$ ) in the subgroups that used dextrose plus icodextrin solutions, or icodextrin solution alone subgroup (subgroup of patients using icodextrin), $24.78 \pm 24.60 \mathrm{~mm}$ in the dextrose solution subgroup (subgroup of patients not using icodextrin); $20.29 \pm 19.72 \mathrm{~mm}$ in the continuous cycling peritoneal dialysis (CCPD) with automated peritoneal dialysis (APD) subgroup; and $27.45 \pm 23.97 \mathrm{~mm}$ in the continuous ambulatory peritoneal dialysis (CAPD; without APD) subgroup. The effect of pruritus-related factors on efficacy was not evaluated.

\section{Safety}

All of the 37 patients were analyzed for safety. The incidence of AEs during clinical trial was 81.1\% (30/37 patients), while that of ADRs was $45.9 \%$ (17/37 patients). The subgroup analysis of different evaluation periods found no increase in AEs or ADRs during the 5- $\mu \mathrm{g}$ administration period. The incidence of AEs and ADRs were, respectively, 51.4\% (19/37 patients) and 35.1\% (13/ 37 patients) in the $2.5-\mu \mathrm{g}$ administration period and $48.6 \%$ (17/35 patients) and $17.1 \%$ (6/35 patients) in the $5-\mu \mathrm{g}$ administration period. Among the ADRs listed in Table 4, the incident rate during treatment period + follow-up period was $5 \%$ or higher for insomnia, somnolence, vomiting, increased blood prolactin, and lower blood testosterone free; all of which were mild. The moderate ADRs occurred in one patient (2.7\%) for drug eruption during the $2.5-\mu \mathrm{g}$ administration period and none at all in the $5-\mu \mathrm{g}$ administration period. None of the ADRs was severe. The difference in PD prescriptions seemed to have minimal effect on the frequency of ADRs. The incidence was $47.1 \%$ (8/17 patients) in the subgroup of patients using icodextrin, 45.0\% (9/20 patients) in the subgroup of patients not using icodextrin, $46.2 \%$ (6/13 patients) in the CCPD subgroup, and $45.8 \%(11 / 24$ patients) in the CAPD subgroup. Laboratory values showed no substantial changes over time. Neither did vital signs nor ECG abnormalities.

\section{Discussion}

Due to the technical need to compare the data obtained in this trial with those from a preceding confirmatory trial in HD patients, virtually the same inclusion and exclusion criteria were set at recruitment so that the characteristics of the patients in the two studies would be as similar as possible. As the equivalence of demographic characteristics was achieved, we decided that it was valid to compare and consider the results of the two studies against each other. 
Table 2 Demographic characteristics and other reference values

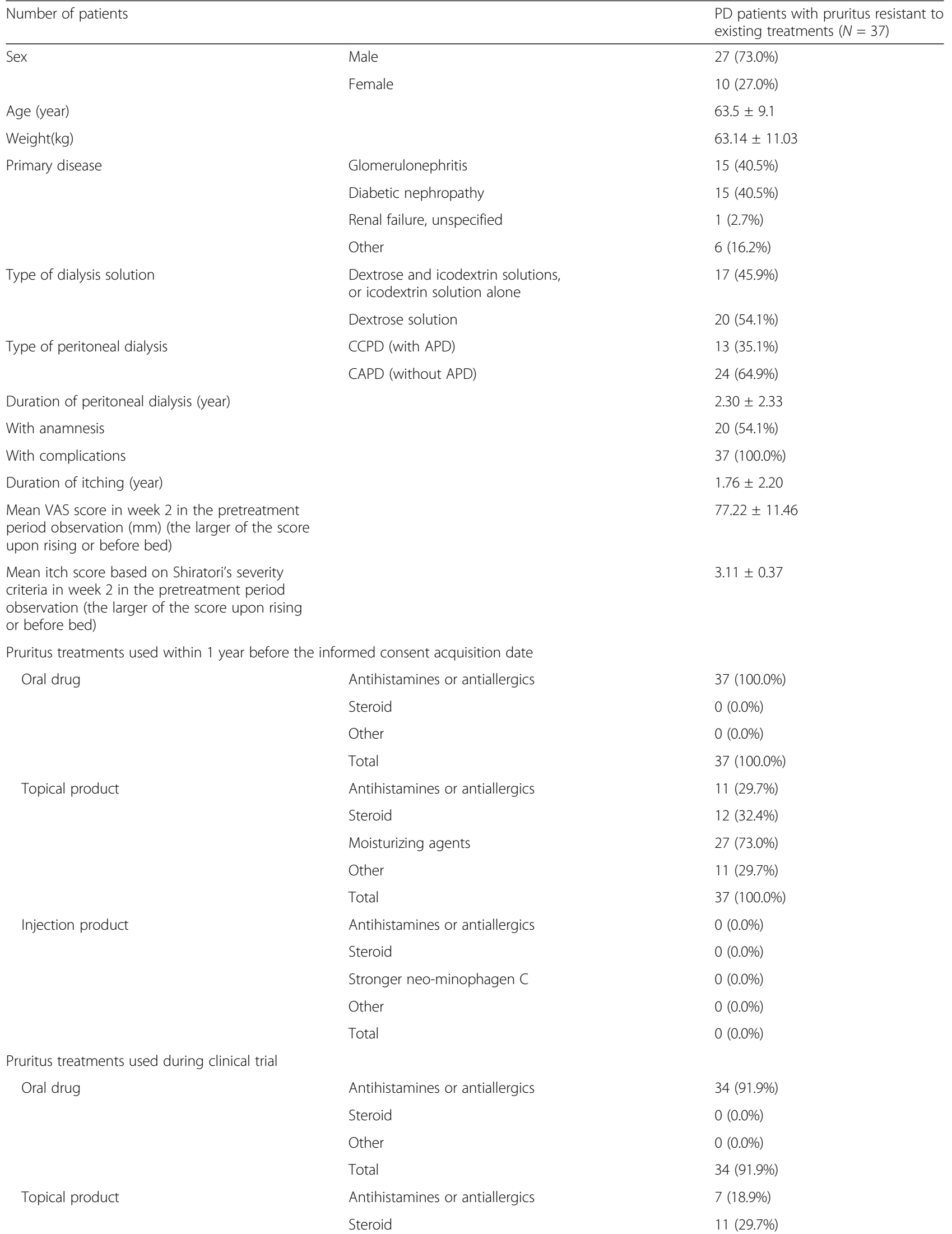


Table 2 Demographic characteristics and other reference values (Continued)

\begin{tabular}{|c|c|c|}
\hline \multicolumn{2}{|l|}{ Number of patients } & \multirow{2}{*}{$\begin{array}{l}\text { PD patients with pruritus resistant to } \\
\text { existing treatments }(N=37) \\
24(64.9 \%)\end{array}$} \\
\hline & Moisturizing agents & \\
\hline & Other & $12(32.4 \%)$ \\
\hline & Total & $37(100.0 \%)$ \\
\hline \multirow[t]{5}{*}{ Injection product } & Antihistamines or antiallergics & $0(0.0 \%)$ \\
\hline & Steroid & $0(0.0 \%)$ \\
\hline & Stronger neo-minophagen $C$ & $1(2.7 \%)$ \\
\hline & Other & $0(0.0 \%)$ \\
\hline & Total & $1(2.7 \%)$ \\
\hline \multicolumn{3}{|l|}{ Pruritus-related factors in CKD patients } \\
\hline \multirow[t]{6}{*}{ Complications (overlapping) } & Hyperphosphatemia & $28(75.7 \%)$ \\
\hline & Secondary hyperparathyroidism & $27(73.0 \%)$ \\
\hline & Asteatosis & $3(8.1 \%)$ \\
\hline & Hypocalcemia & $1(2.7 \%)$ \\
\hline & Xeroderma & $1(2.7 \%)$ \\
\hline & Immunodeficiency & $1(2.7 \%)$ \\
\hline \multirow[t]{8}{*}{ Laboratory value (pretreatment value) } & Mean plasma hemoglobin (g/dL; \pm SD) & $10.58 \pm 1.33$ \\
\hline & Mean plasma eosinophils (\%; \pm SD) & $7.43 \pm 6.54$ \\
\hline & Mean serum albumin (g/dL; \pm SD) & $3.49 \pm 0.40$ \\
\hline & Mean plasma hematocrit (\%; \pm SD) & $31.98 \pm 4.22$ \\
\hline & Mean serum urea nitrogen $(\mathrm{mg} / \mathrm{dL} ; \pm \mathrm{SD})$ & $56.2 \pm 14.1$ \\
\hline & Mean serum creatinine $(\mathrm{mg} / \mathrm{dL} ; \pm \mathrm{SD})$ & $9.953 \pm 3.179$ \\
\hline & Mean serum phosphorus (mg/dL; $\pm S D)$ & $5.18 \pm 1.10$ \\
\hline & Mean serum calcium (mg/dL; $\pm S D$ ) & $8.85 \pm 0.89$ \\
\hline
\end{tabular}

Values are the number (percentage) of cases

$C C P D$ continuous cycling peritoneal dialysis, $C A P D$ continuous ambulatory peritoneal dialysis, $A P D$ automated peritoneal dialysis, VAS visual analog scale, $C K D$ chronic kidney disease, $S D$ standard deviation

The VAS change observed in this trial was equivalent to that in the preceding confirmatory trial; the VAS change (LOCF) in week 2 of the treatment period in this trial was $24.93 \pm 22.56 \mathrm{~mm}$ (mean $\pm \mathrm{SD}$ ), compared to the primary endpoint (week 2 in the $2.5 \mu \mathrm{g}$ group) of $24.52 \pm 21.82 \mathrm{~mm}$ in the confirmatory trial in $\mathrm{HD}$ patients. Moreover, the present trial found the change over time in the mean VAS change, VAS improvement, and the course of changes in the mean pruritus score to be similar between the HD and PD patient groups. Thus, the present trial demonstrated the clinical effectiveness of nalfurafine $2.5 \mu \mathrm{g}$ for the treatment of pruritus resistant to existing treatments in PD patients. Furthermore, subgroup analyses found no noticeable effect on efficacy of different prescriptions of PD. In terms of different methods of PD, the change in VAS was no difference between the subgroup with APD and without APD; additionally, the use of different dialysate solutions did not result in significantly different VAS changes. This trial also suggested that the efficacy of nalfurafine $5 \mu \mathrm{g}$ in
PD patients was virtually the same as in HD patients. The VAS changes in weeks 3 and 4 in the treatment period of this trial, when participants received nalfurafine $5 \mu \mathrm{g}$ $(28.94 \pm 22.01 \mathrm{~mm}$ in week $3,32.13 \pm 23.44 \mathrm{~mm}$ in week 4), were found to be equivalent to the $28.28 \pm 23.14 \mathrm{~mm}$ VAS change observed in the $5 \mu \mathrm{g}$ group in week 4 in the treatment period of a preceding long-term trial in HD patients [7].

Safety assessment showed that nalfurafine $2.5 \mu \mathrm{g}$ or $5 \mu \mathrm{g}$ in PD patients is well tolerated. No additional incidents of ADRs were observed in the 5- $\mu \mathrm{g}$ dosage period over the $2.5-\mu \mathrm{g}$ dosage period. Most of the ADRs that did occur during this trial were mild; none was serious. The safety of nalfurafine according to different dialysis methods was also examined by comparing the incidents of ADRs in this trial with those in the preceding confirmatory trial in HD patients. Neither the incident rate of ADRs nor of serious drug reactions or moderate or worse drug reactions in PD patients was significantly higher than 

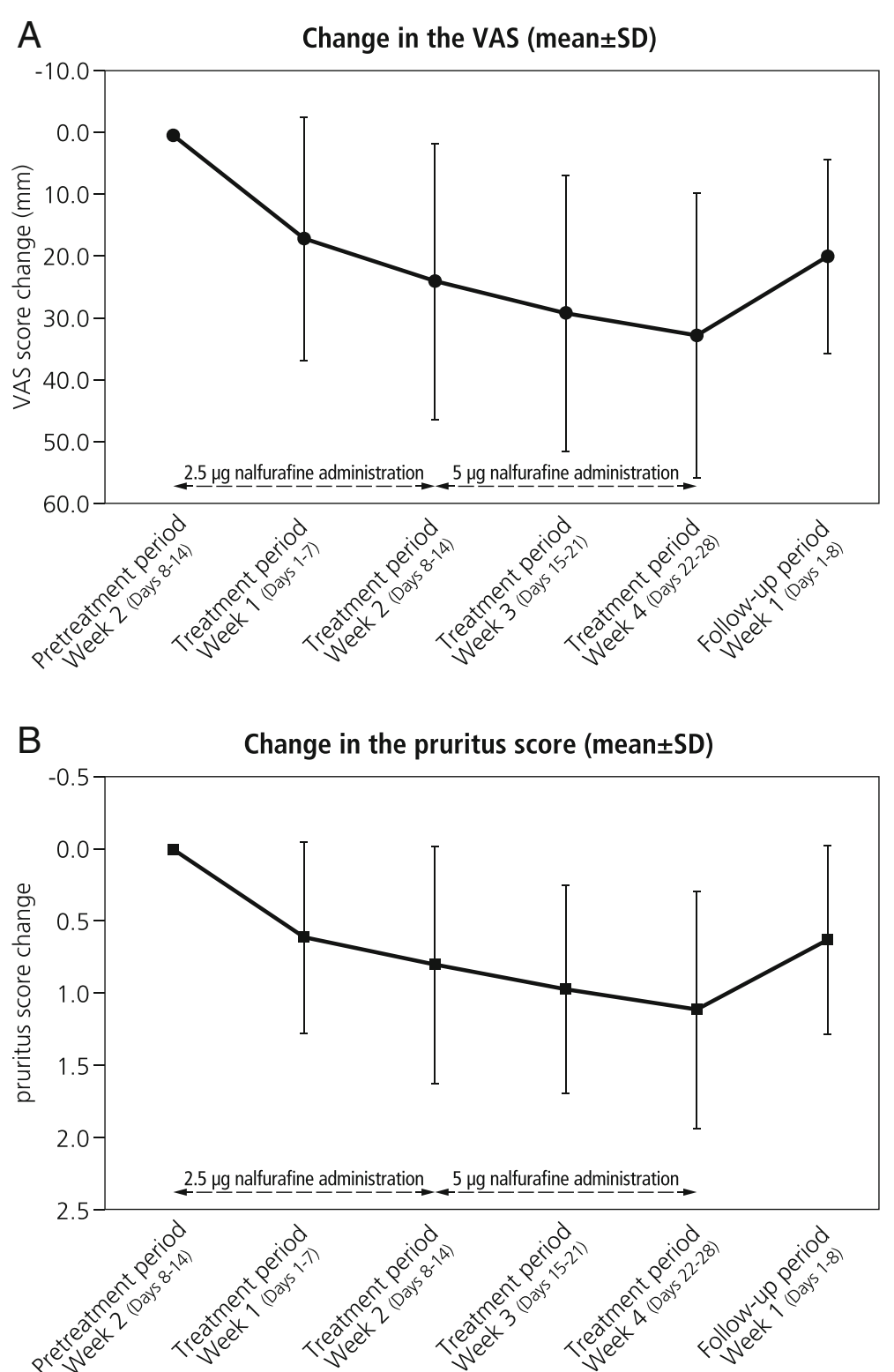

Fig. 2 The courses of the VAS value and the pruritus score changes (FAS). a shows the change in the VAS, while $\mathbf{b}$ shows that in the pruritus score. (mean \pm SD)

in HD patients. Although PD patients reported relatively higher incidence of somnolence, vomiting, increased blood prolactin, and lower blood testosterone free than in HD patients, these ADRs were all mild and resolved without any treatment; none was serious. The effect of nalfurafine on the endocrine system has been confirmed in previous non-clinical and clinical trials. The agent appears to induce endocrinal changes by actions common to those of opioids, which are known to act on the central nervous system and may trigger endocrinal disorders [15]. Increased blood prolactin and lower blood

Table 3 VAS improvement (FAS)

\begin{tabular}{|c|c|c|c|c|c|}
\hline & $\begin{array}{l}\text { Treatment } \\
\text { period week } 1 \\
\text { (days } 1-7) \\
(N=37)\end{array}$ & $\begin{array}{l}\text { Treatment } \\
\text { period week } 2 \\
\text { (days } 8-14) \\
(N=36)\end{array}$ & $\begin{array}{l}\text { Treatment } \\
\text { period week } 3 \\
\text { (days 15-21) } \\
(N=35)\end{array}$ & $\begin{array}{l}\text { Treatment } \\
\text { period week } 4 \\
\text { (days 22-28) } \\
(N=35)\end{array}$ & $\begin{array}{l}\text { Follow-up } \\
\text { period week } 1 \\
\text { (days 1-8) } \\
(N=36)\end{array}$ \\
\hline Improved patients (\%) [95\% Cl] & $11(29.7)[15.9,47.0]$ & $18(50.0)[32.9,67.1]$ & $22(62.9)[44.9,78.5]$ & $22(62.9)[44.9,78.5]$ & $19(52.8)[35.5,69.6]$ \\
\hline
\end{tabular}


Table 4 The list of adverse drug reactions

\begin{tabular}{|c|c|c|c|c|}
\hline & $\begin{array}{l}2.5-\mu \mathrm{g} \text { administration } \\
\text { period }\end{array}$ & $\begin{array}{l}5-\mu g \text { administration } \\
\text { period }\end{array}$ & $\begin{array}{l}5-\mu g \text { administration } \\
\text { period + follow-up period }\end{array}$ & $\begin{array}{l}\text { Treatment period } \\
+ \text { follow-up period }\end{array}$ \\
\hline & $N=37$ & $N=35$ & $N=35$ & $N=37$ \\
\hline Adverse drug reactions & Number of patients (\%) & Number of patients (\%) & Number of patients (\%) & Number of patients (\%) \\
\hline Insomnia & $3(8.1)$ & $2(5.7)$ & $2(5.7)$ & $5(13.5)$ \\
\hline Dizziness & $1(2.7)$ & $0(0.0)$ & $0(0.0)$ & $1(2.7)$ \\
\hline Hypoaesthesia & $1(2.7)$ & $0(0.0)$ & $0(0.0)$ & $1(2.7)$ \\
\hline Somnolence & $3(8.1)$ & $0(0.0)$ & $0(0.0)$ & $3(8.1)$ \\
\hline Restless legs syndrome & $0(0.0)$ & $1(2.9)$ & $1(2.9)$ & $1(2.7)$ \\
\hline Constipation & $1(2.7)$ & $0(0.0)$ & $0(0.0)$ & $1(2.7)$ \\
\hline Gastrooesophageal reflux disease & $0(0.0)$ & $1(2.9)$ & $1(2.9)$ & $1(2.7)$ \\
\hline Vomiting & $1(2.7)$ & $1(2.9)$ & $1(2.9)$ & $2(5.4)$ \\
\hline Hypoaesthesia oral & $1(2.7)$ & $0(0.0)$ & $0(0.0)$ & $1(2.7)$ \\
\hline Hepatic function abnormal & $1(2.7)$ & $0(0.0)$ & $0(0.0)$ & $1(2.7)$ \\
\hline Drug eruption & $1(2.7)$ & $0(0.0)$ & $0(0.0)$ & $1(2.7)$ \\
\hline Pruritus & $1(2.7)$ & $0(0.0)$ & $0(0.0)$ & $1(2.7)$ \\
\hline Rash & $1(2.7)$ & $0(0.0)$ & $0(0.0)$ & $1(2.7)$ \\
\hline Haematuria & $1(2.7)$ & $0(0.0)$ & $0(0.0)$ & $1(2.7)$ \\
\hline Asthenia & $1(2.7)$ & $0(0.0)$ & $0(0.0)$ & $1(2.7)$ \\
\hline Feeling hot & $1(2.7)$ & $0(0.0)$ & $0(0.0)$ & $1(2.7)$ \\
\hline Aspartate aminotransferase increased & $0(0.0)$ & $0(0.0)$ & $1(2.9)$ & $1(2.7)$ \\
\hline Blood prolactin increased & $2(5.4)$ & $2(5.7)$ & $3(8.6)$ & $5(13.5)$ \\
\hline Blood testosterone free decreased & $2(5.4)$ & $1(2.9)$ & $1(2.9)$ & $3(8.1)$ \\
\hline
\end{tabular}

testosterone free observed in the present trial were only temporary changes. No endocrine-related clinical symptoms such as cardiovascular diseases or death were observed. These findings indicate that PD patients are very unlikely to experience clinically significant ADRs of nalfurafine, and that no special caution needs to be applied to those existing for use of nalfurafine in HD patients.

While HD is highly standardized, PD is diverse in terms of the choice and adjustment of the type of PD, the method of exchanging dialysate, and the dialysis dose which is tailored to each patient's physical status and lifestyle. After due consideration of adaptation to the specifically prescribed approach, it was decided that the results of a previous trial rejected the need for adjustment of the dosage and administration of nalfurafine according to different prescriptions of PD. In a preceding clinical pharmacology trial, $17 \mathrm{PD}$ patients treated with a single oral dose of nalfurafine $2.5 \mu \mathrm{g}$ or $5 \mu \mathrm{g}$ had been examined for how different prescriptions of PD potentially affected nalfurafine pharmacokinetics. The trial found that neither the method of PD nor the type of dialysate affected nalfurafine pharmacokinetics (unpublished observations). The results were further reinforced by the present trial as detailed above in the efficacy and safety sections.
Overall, the available information suggests that PD is similar to HD in requiring no adjustment of dosage or administration. The pharmacokinetics of nalfurafine in the $17 \mathrm{PD}$ patients in the preceding trial was compared with that in $16 \mathrm{HD}$ patients who were enrolled in another clinical pharmacological trial. This comparison revealed the pharmacokinetics of nalfurafine in the two groups to be very similar.

Nalfurafine is approved in Japan and Korea for the treatment of intractable pruritus in HD patients, and two dosages of $2 \mu \mathrm{g}$ and $5 \mu \mathrm{g}$ have been in clinical use. In addition, Takahashi and others [16] have proposed a treatment algorithm of considering elevation to $5 \mu \mathrm{g}$ if sufficient efficacy is not achieved after administering $2.5 \mu \mathrm{g}$ for 2 to 4 weeks. Therefore, as in HD patients, the efficacy of nalfurafine for treating refractory pruritus in $\mathrm{PD}$ patients should be evaluated after administering $2.5 \mu \mathrm{g} /$ day for 2 to 4 weeks. If the efficacy is insufficient without troublesome ADRs, elevation to $5 \mu \mathrm{g} /$ day can be considered.

The limitations of this clinical trial include its openlabel design, the use of short administration periods, and 2.5- $\mu \mathrm{g}$ and 5- $\mu \mathrm{g}$ nalfurafine dosage variation that did not allow adequate assessment of the effect of treatment for the pruritus of PD patients. Nevertheless, this trial is 
notable as the first study showing the efficacy and safety of nalfurafine in PD patients with pruritus resistant to existing treatments.

\section{Conclusions}

Nalfurafine at $2.5 \mu \mathrm{g}$ and $5 \mu \mathrm{g}$ may be effective for the treatment of refractory pruritus in patients undergoing PD as well as in HD patients. Since the safety profile of nalfurafine in PD patients was very similar to that in HD patients, we determined that the dosage and administration in PD patients can be the same as in HD patients; starting at $2.5 \mu \mathrm{g}$, the clinical dosage may be increased up to $5 \mu \mathrm{g}$ according to prevailing symptoms. No adjustment of dosage and administration seems necessary relative to various $\mathrm{PD}$ prescriptions.

\begin{abstract}
Abbreviations
ADR: Adverse drug reaction; APD: Automated peritoneal dialysis; CAPD: Continuous ambulatory peritoneal dialysis; CCPD: Continuous cycling peritoneal dialysis; Cl: Confidence interval; ECG: Electrocardiogram; ESRD: End-stage renal disease; FAS: Full analysis set; IgA: Immunoglobulin A J-GCP: Japanese Good Clinical Practice; LOCF: Last observation carried forward; OTC: Over-the-counter; SD: Standard deviation; VAS: Visual analog scale
\end{abstract}

\section{Acknowledgements}

We would like to express our gratitude to all patients registered in this clinical trial, all participating institutions, their principal and other investigators, and clinical research staff.

\section{Funding}

The financial cost to conduct this clinical trial was covered by its sponsor, Toray Industries, Inc. English translation was prepared by Jun Kishi of Medcore Associates, Inc. and Dr. Martin Guppy of Metamols Ltd.

\section{Availability of data and materials}

The datasets generated and/or analyzed during the current trial are available from the corresponding author on reasonable request.

\section{Authors' contributions}

HN provided medical advice in creating and amending the protocol. Receiving the information on safety, he provided medical advice when appropriate. He discussed each case and its handling in accordance with the GCPs with the sponsor. He read and signed the clinical study report and wrote this manuscript. TO, SM, and El are responsible for the general administration and management of the clinical trial. They created and amended the protocol and responded to issues during the clinical trial. They gave instructions for the preparation of the clinical study report and assume responsibility for its content. MS analyzed the pharmacokinetics of the investigational drug in a peritoneal dialysis patient. All authors have read and approved the present paper and consented to its submission.

\section{Ethics approval and consent to participate}

This clinical trial was approved by each of all the institutional review boards of participating institutions and conducted in compliance with the ethical principles of the Declaration of Helsinki, the Pharmaceutical and Medical Device Act, and the Ministerial Ordinance on Good Clinical Practice for Drugs (J-GCP). Written informed consent was obtained from all participating patients.

\section{Consent for publication}

Not applicable

\section{Competing interests}

This clinical trial was funded by Toray Industries, Inc. HN received consulting fee from Toray Industries, Inc. TO, MS, El, and SM are employees of Toray Industries, Inc. No other potential competing interest relevant to this article was reported.

\section{Publisher's Note}

Springer Nature remains neutral with regard to jurisdictional claims in published maps and institutional affiliations.

\section{Author details}

${ }^{1}$ Department of General Internal Medicine, Saitama Medical University Hospital, 38 Morohongo, Moroyama-machi, Iruma-gun, Saitama 350-0495, Japan. ${ }^{2}$ Pharmaceutical Clinical Research Department, Toray Industries Inc., 1-1, Nihonbashi-muromachi 2-chome, Chuo-ku, Tokyo 103-8666, Japan. ${ }^{3}$ Pharmaceuticals Business Department, Toray International (China) Co., Ltd, 1601 West Nanjing Road, Jing An District, Shanghai 200040, China.

Received: 30 May 2017 Accepted: 20 September 2017

Published online: 15 December 2017

\section{References}

1. Tessari G, Dalle Vedove C, Loschiavo C, Tessitore N, Rugiu C, Lupo A, et al. The impact of pruritus on quality of life of patients undergoing dialysis: a single centre cohort study. J Nephrol. 2009;22:241-8.

2. Narita I, Iguchi S, Omori K, Gejyo F. Uremic pruritus in chronic hemodialysis patients. J Nephrol. 2008;21:161-5.

3. Krajnik M, Zylicz Z. Understanding pruritus in systemic disease. J Pain Symptom Manag. 2001;21:151-68.

4. Togashi $Y$, Umeuchi $H$, Okano $K$, Ando N, Yoshizawa $Y$, Honda $T$, et al. Antipruritic activity of the $\mathrm{K}$-opioid receptor agonist, TRK-820. Eur J Pharmacol. 2002:435:259-64.

5. Umeuchi H, Togashi Y, Honda T, Nakao K, Okano K, Tanaka T, et al. Involvement of central $\mu$-opioid system in the scratching behavior in mice, and the suppression of it by the activation of k-opioid system. Eur J Pharmacol. 2003;477:29-35.

6. Kumagai $H$, Ebata T, Takamori $K$, Muramatsu T, Nakamoto H, Suzuki H. Effect of a novel Kappa-receptor agonist, nalfurafine hydrochloride, on severe itch in 337 haemodialysis patients: a phase III, randomized, double-blind, placebo-controlled study. Nephrol Dial Transplant. 2010;25:1251-7.

7. Kumagai H, Ebata T, Takamori K, Miyasato K, Muramatsu T, Nakamoto H, et al. Efficacy and safety of a novel $\mathrm{k}$-agonist for managing intractable pruritus in dialysis patients. Am J Nephrol. 2012;36:175-83.

8. Kumada H, Miyakawa H, Muramatsu T, Ando N, Oh T, Takamori K, et al. Efficacy of nalfurafine hydrochloride in patients with chronic liver disease with refractory pruritus: a randomized, double-blind trial. Hepatol Res. 2017; 47:972-82.

9. Mistik S, Utas S, Ferahbas A, Tokgoz B, Unsal G, Sahan H, et al. An epidemiology study of patients with uremic pruritus. J Eur Acad Dermato. 2006:20:672-8.

10. Takamori $\mathrm{K}$, et al. Role of the opioid system in systemic and peripheral itch. $20^{\text {th }}$ World Congress of Dermatology. Paris, France 2002.

11. An overview of regular dialysis treatment in Japan as of Dec. 31, 2015 Japanese Society for Dialysis Therapy. http://docs.jsdt.or.jp/overview/ pdf2016/p042.pdf. Accessed 1 Aug 2017.

12. Wahlgren CF, Ekblom A, Hägermark O. Some aspects of the experimental induction and measurement of itch. Acta Derm Venereol. 1989;69:185-9.

13. Kawashima M, Tango $T$, Noguchi $T$, Inagi M, Nakagawa $H$, Harada S. Addition of fexofenadine to a topical corticosteroid reduces the pruritus associated with atopic dermatitis in a 1-week randomized, multicentre, double-blind, placebo-controlled, parallel-group study. Br J Dermatol. 2003;148:1212-21.

14. Hiraga K, Ohashi Y. Efficacy evaluation of analgesic agents used for cancer pain management by visual analogue scale-a survey by questionnaire to physicians, nurses and patients. Pain Res. 1999:14:9-19.

15. Grossman A. Brain opiates and neuroendocrine function. Clin Endocrinol Metab. 1983:12:725-46.

16. Takahashi N, Yoshizawa T, Kumagai J, Kawanishi H, Moriishi M, Masaki T, et al. Response of patients with hemodialysis-associated pruritus to new treatment algorithm with nalfurafine hydrochloride: a retrospective survey-based study. Renal Replacement Therapy. 2016;2:27. 